\title{
Article
}

\section{The influence of coach turnover on student-athletes' affective states and team dynamics: An exploratory study in collegiate sports}

Shipherd, Amber M., Wakefield, Joann C., Stokowski, Sarah and Filho, Edson

Available at http://clok.uclan.ac.uk/22103/

Shipherd, Amber M., Wakefield, Joann C., Stokowski, Sarah and Filho, Edson ORCID: 0000-0002-8548-4651 (2019) The influence of coach turnover on student-athletes' affective states and team dynamics: An exploratory study in collegiate sports. International Journal of Sports Science \& Coaching, 14 (1). pp. 97-106. ISSN 1747-9541

It is advisable to refer to the publisher's version if you intend to cite from the work. http://dx.doi.org/10.1177/1747954118766465

For more information about UCLan's research in this area go to http://www.uclan.ac.uk/researchgroups/ and search for <name of research Group>.

For information about Research generally at UCLan please go to http://www.uclan.ac.uk/research/

All outputs in CLoK are protected by Intellectual Property Rights law, including Copyright law. Copyright, IPR and Moral Rights for the works on this site are retained by the individual authors and/or other copyright owners. Terms and conditions for use of this material are defined in the policies page. 
INFLUENCE OF COACH TURNOVER ON STUDENT-ATHLETES

The Influence of Coach Turnover on Athletes' Affective States and Team Dynamics: An Exploratory Study in Collegiate Sports

Amber M. Shipherd ${ }^{1}$, Joann C. Wakefield ${ }^{2}$, S. E. Stokowski ${ }^{3}, \&$ Edson Filho $^{4 *}$

${ }^{1}$ Department of Health and Kinesiology, Texas A\&M University - Kingsville

${ }^{2}$ School of Health and Kinesiology, Georgia Southern University

${ }^{3}$ Department of Health, Human Performance, and Recreation, University of Arkansas

${ }^{4}$ School of Psychology, University of Central Lancashire

\section{*Corresponding Author:}

Edson Filho, PhD.

Lecturer (Assistant Professor) in Sport and Exercise Psychology

School of Psychology

University of Central Lancashire

Darwin Building 114

Preston PR1 2HE

United Kingdom

Tel: +44 (0) 1772893436

Email: efilho@uclan.ac.uk 
INFLUENCE OF COACH TURNOVER ON STUDENT-ATHLETES

\begin{abstract}
Coach turnover is frequent, especially among collegiate sports in the United States. To date, however, there is scant research on how coach turnover might affect student-athletes. Accordingly, we aimed to qualitatively explore how coach turnover might influence studentathletes' psychosocial states and team dynamics in collegiate sports. Eleven athletic teams at a Midwestern Division I NCAA institution having undergone coach turnover during the past four years participated in the study. Four coaches and 21 student-athletes representing both individual and team sports, as well as two administrative staff members, participated in semi-structured interviews (coaches and administrators) and focus groups (student-athletes). Inductive data analysis revealed four first level themes: coach style and background, athlete affective states, team dynamics, and program culture. Our analysis suggested that, depending on their gender and personal characteristics, coaches' might have a positive or negative impact on athletes' affective states (e.g., positive and negative affect), team dynamics (e.g., cohesion; athlete leadership), and the environment at large (e.g., athletic program). Thus, practitioners might facilitate the positive impact of coaching turnover by assessing whether the new coach is a "good fit" at the individual (micro), team (meso), and environmental (macro) level of analysis.
\end{abstract}

Keywords: Coach turnover, student-athletes, collegiate sports, career transition, group dynamics. 
INFLUENCE OF COACH TURNOVER ON STUDENT-ATHLETES

The Influence of Coach Turnover on Student-Athletes' Affective States and Team Dynamics:

An Exploratory Study in Collegiate Sports

According to the National Collegiate Athletic Association (NCAA; 2011) nearly half of intercollegiate football student-athletes selected their respective institutions based solely on the coach. Coaches play an important role in the development of student-athletes, undertaking the roles of teachers and mentors (Brubaker, 2007; Lattman, 2008). Although coaches assist in the overall growth of student-athletes, many coaches will leave the athletes they recruited due to lack of athletic success or perhaps to pursue other coaching opportunities (Carron \& Eys, 2012). The study proposed herein stems from data suggesting that many colleges and universities in the United States experience high coach turnover (Winthrop, 2013).

Coaching turnovers are not only costly (Mitchell, Holtom, \& Lee, 2001) but also carry broader implications for sports program and athletic departments (Cunningham, Sagas, \& Ashley, 2001; Ryan \& Sagas, 2009). Coaches are vital to the athletic culture and "sport organizations recognize that continuity among coaches is important to sustain quality sport programs" (Raedeke, Warren, \& Granzke, 2002, p. 73). In many cases, coaches resign or are terminated from an institution due to underperformance (Carron \& Eys, 2012; White, Persad, \& Gee, 2007), and new coaches are often hired with the intention to increase success (Colvin et al., 2012; Fabianic, 1994; White et al., 2007). Similar to the White et al. (2007) study, a longitudinal study by Humphreys, Paul, and Weinbach (2016) showed that football coaching tenure at NCAA Division I institutions were contingent upon not only team successes on the field but myriad other expectations, such as rankings, quality of recruiting class, and conference championships.

While research indicates that coach turnover can have a negative impact on studentathletes academically (e.g., Johnson et al., 2013; Johnson, Wessel, \& Pierce, 2012), there is a gap 
INFLUENCE OF COACH TURNOVER ON STUDENT-ATHLETES

in the literature on how coach turnover affects student-athletes' psychosocial states. Previous research has shown that coach behaviors influence student outcomes and social variables across several domains of human performance (Côté \& Gilbert, 2009; Gershgoren, Filho, Tenenbaum, \& Schinke, 2013). The importance of the coach-athlete relationship is well documented (e.g., Jackson, Knapp, \& Beauchamp, 2009; Jowett, 2009; Jowett \& Ntoumanis, 2004; Jowett, Paull, \& Pensgaard, 2005). A healthy relationship between a coach and an athlete can have a positive impact on individuals (e.g., increased self-esteem and satisfaction; Jowett, 2005), as well as improve team dynamics (Shipherd, Basevitch, Barcza-Renner, \& Siwatu, 2014; Turman, 2008). More specifically, coaches cultivating a mastery-oriented climate will support the development of athlete efficacy beliefs, which results in athletes experiencing positive affective states (Braithwaite, Spray, \& Warburton, 2011). On the other hand, a negative coach-athlete relationship may lead to athletes experiencing adverse cognitive and affective states, as well as dysfunctional behavioral patterns both on and off the field. Coaches who foster an ego-oriented climate are more likely to observe negative affective states with their athletes (Duda, Papaioannou, Appleton, Quested, \& Krommidas, 2014).

Despite the role that coaches play in athlete development, coach turnover does not always have a negative impact on those involved. Research supports that if conducted in a certain manner, a coach turnover can have positive consequences for both the athletes and the team as a whole. A study in swimming revealed that current coaches, compared to former coaches, reported having higher investment and commitment to their coaching job (Raedeke et al., 2002). Another study by White et al. (2007) examined how four mid-season coaching changes influenced performance in team sports and revealed that, although the teams did not experience immediate success, team performance increased in the following season. Therefore, the best time 
INFLUENCE OF COACH TURNOVER ON STUDENT-ATHLETES

for a coaching change is probably during the off-season (Audas, Dobson, \& Goddard, 2002;

White et al., 2007). White et al. (2007) observed that coaching changes that occur in the offseason are more likely to be successful because the incoming coach and current athletes have time to build rapport. These findings, alongside Colvin et al. (2012), suggest that perhaps coach turnover can be positive in regards to the possibility of a new coach "turning around" a program by improving not only the winning record but also the overall motivational climate in the team.

Noteworthy, the motivational climate established by coaches influence how athletes think, feel, and act (e.g., the cognitive-affective-behavioral link; see Tenenbaum, Basevitch, Gershgoren, \& Filho, 2013). To this extent, achievement goal theory has been used as a framework for studying the coach-athlete relationship and forms the theoretical ground of the present study (Nicholls, 1992). Previous empirical evidence corroborates achievement goal theory tenets in which a predominantly task-oriented climate increases the chances of individuals experiencing positive affect and intra-group dynamics, whereas a predominantly ego-oriented climate increases the chances of athletes experiencing negative affect and intra-group conflicts (Bortoli, Bertollo, Comani, \& Robazza, 2011; Bortoli, Bertollo, Filho, \& Robazza, 2014). However, previous research in this area has not looked at the influence of coach turnover per se but rather has focused on how current coaches might influence athletes' psychosocial states in particular, and group dynamics at large (Duda et al., 2014).

Indeed, coach turnover studies have focused primarily on the coach, thus failing to recognize the needs of the athletes who have just experienced a change in leadership (Pate, Stokowski, \& Hardin, 2011). Accordingly, in the present study we aimed to explore how coach turnover in collegiate athletics might influence athletes' affective states and team dynamics. This purpose was addressed through two research questions: (1) How does coach turnover in 
INFLUENCE OF COACH TURNOVER ON STUDENT-ATHLETES

collegiate sports influence student-athletes' affective states? and (2) How does coach turnover in collegiate sports influence team dynamics?

\section{Methods}

\section{Design}

An interpretative phenomenological analysis (IPA) approach was deemed the most appropriate methodology for this study. Phenomenological approaches study individuals' subjective experiences through rich descriptions and narratives from participants (Davidsen, 2013). IPA studies, a particular phenomenological approach, aim to explore how a specific group of participants interpret and make sense of both their personal and social worlds (Smith \& Osborn, 2015). In particular, IPA studies examine and attempt to understand individuals' personal experience of an event. This methodological approach is commonly used in psychological research considering the emphasis on "sense-making," meaning it is concerned with both cognitive and social processes (Smith \& Osborn, 2015). Given IPA's focus on participants' experiences and interpretation of those experiences resulting from an individuals' social world, IPA is considered to be based in constructivism, or the idea that learners construct knowledge and meaning from their own experiences (Davidsen, 2013). An IPA approach was appropriate for this study as the primary objective was to gain a better understanding of the psychosocial experiences of student-athletes who had undergone a coaching change. Additionally, given that all of the participants worked at or attended the same university, this study can also be considered to be a case study in design.

\section{Participants}

After obtaining Institutional Review Board approval, all athletic teams having undergone coach turnover during the past four years at a Midwestern Division I NCAA institution were 
INFLUENCE OF COACH TURNOVER ON STUDENT-ATHLETES

contacted to participate in the study. Nine new (just hired) coaches representing 11 teams meeting the study inclusion criteria were recruited via email and were asked to forward the email to eligible junior or senior student-athletes on their team. Two athletics department administrative staff members were also contacted via email to participate in interviews. The recruiting email asked participants to contact the first author to arrange a day and time to participate in either an interview (coaches and athletics staff) or a small focus group (athletes). The researchers utilized pseudonyms and changed the specific sports to ensure the anonymity of the participants.

A total of four new coaches, 21 student-athletes, and two administrative staff members participated in this study. The student-athletes participated in both independent (e.g., swimming) and interdependent (e.g., soccer) sports, and reported having between 10 and 19 years of experience competing in their sports $(M=15.24 ; \mathrm{SD}=2.81)$. Six student-athletes held formal leadership roles on their team (e.g., captain). The coaches also represented independent and interdependent teams, and reported having between 16 and 20 years of coaching experience in their sport $(M=18 ; \mathrm{SD}=2.19)$, and between one semester and two years of experience in their current position $(M=13.17$ months; $\mathrm{SD}=8.95)$. The two administrative staff participants from the athletics department reported having eight and ten years of experience working in athletics, respectively.

\section{Procedures}

The researchers collected data via four individual coach interviews, two individual interviews with athletics administrative staff, 11 focus groups and interviews with studentathletes, and analyzed one newspaper article. At the beginning of each interview or focus group, participants were asked to read and sign the informed consent if they agreed to participate in the 
INFLUENCE OF COACH TURNOVER ON STUDENT-ATHLETES

study. The interviews and focus groups were led by the primary investigator, were audio recorded to ensure accuracy of information, and lasted between thirteen and thirty-four minutes $(M=19 \min 58 \mathrm{~s} ; S D=3 \min 49 \mathrm{~s})$. The interviews began with a few brief demographic questions (e.g., how long they have been playing or coaching their sport) to establish rapport (Patton, 2002). Subsequently, participants were asked two core open-ended questions regarding the focus areas of this study: student-athlete affective states and team dynamics. For example, "Describe how it has been like for you and your team since you had a head coaching change." Some participants were asked follow-up questions as needed, such as, "Can you give an example of how your team became closer through the coaching change?"

\section{Data Analysis}

The data analysis began with verbatim data transcription of the interviews and focus groups. The researchers analyzed the transcripts and newspaper article using the constant comparative method (Glaser, 1985). Data coding consisted of identifying and separating text into meaning units (MUs). MUs were then examined for similarities and differences and separated into themes (Teddlie \& Tashakkori, 2009). This process continued until all text segments were grouped into themes with maximum between-theme variation and minimum within-theme variation. Once no new themes emerged, the researchers assumed saturation.

Trustworthiness was established through member checking, triangulation of data sources, and investigator triangulation (Teddlie \& Tashakkori, 2009). The participants received the verbatim transcripts of their interviews and were asked to verify the accuracy of the data. Participants were asked to contact the first author with any general feedback or if they believed there were any discrepancies between their intended meanings and what was transcribed. None of the participants responded to the request, indicating that either the participants noted no 
INFLUENCE OF COACH TURNOVER ON STUDENT-ATHLETES

discrepancies or that they lacked interest or motivation to read the transcript or respond back.

Data were also collected from multiple coaches, student-athletes, and athletics administrative personnel to triangulate the data sources.

Finally, for investigator triangulation, two of the researchers independently analyzed the data, then discussed and agreed on the themes. The independent analyses revealed a high degree of consistency in regards to theme development $(\mathrm{K}=.94, \mathrm{p}<.001)$. Any disagreements were resolved by the researchers who acted as critical friends to one another until consensus about the coding for all themes and sub-themes was reached. Once these themes and MUs were agreed upon by three of the researchers, representative quotes were selected to illustrate each secondlevel theme, and a conceptual map showing the relationship amongst themes and sub-themes was developed.

\section{Results}

We identified four first-level themes representing the micro (i.e., coaches' characteristics and athletes' affective states), meso (i.e., team dynamics) and macro (i.e., program culture) levels of analysis, as well as nine second-level themes as visually depicted in Figure 1. These themes and sub-themes are described below.

\section{Coaches' Characteristics}

Several themes emerged from the data specific to the new coach that influenced the quality of the turnover. More specifically, participants discussed how the new coach's style of coaching, as well the new coach's gender, as being integral to the quality of the coach turnover.

Gender. The coaches' gender seemed to have played an important role in the quality of the coach turnover. When the new coach's gender was the same as the athletes', the result 
INFLUENCE OF COACH TURNOVER ON STUDENT-ATHLETES

seemed to be a more positive transition. For example, Michelle was a female coach of the women's tennis team, whose previous coach was male. Michelle commented:

I think actually having a female coach just did wonders for them umm...and not only myself but we had [the team's female graduate assistant] last year. We have [another female graduate assistant] this year...like there's not many Division I programs that have a female staff, if any. Umm... so I think that just did a lot more than I think I want to realize at times.

Michelle went on to add that, “[confidence] was my biggest goal of the women's team that you gain confidence in your team, your [playing], yourself...all of these things and they've definitely grown in that." However, when the new coach's gender was different from the athletes', the transition appeared to have more of a negative impact. Jon, a male tennis player, reported:

There's been a lot more backlash with our current coach than there was the previous and I think it comes down to her being a female... and I think it's just the stereotype across all sports teams. That the stereotype is to have a male coach rather than a female coach and like...I don't like, I personally don't have a problem with having a female coach because I grew up having male and female coaches but there are people on the team who strictly come from an all male coaching background, so like transitioning from a male to a female took an impact on how they saw things and they didn't believe that she knew what she was doing.

Coaching style. Another theme that emerged and likely influenced the quality of the coach turnover was the new coach's style. In particular, coaches who implemented changes to 


\section{INFLUENCE OF COACH TURNOVER ON STUDENT-ATHLETES}

the team at a slower pace, rather than all at once, experienced a more positive coach turnover. Byron, an administrator, said:

There's things that [the new coach] didn't like about the way things were done but she didn't change them because it wasn't negative. Our line-ups, like people own their spot in their line up and that doesn't necessarily help win matches sometimes. So this year she kind of let them have their own spots. This last weekend, starting this weekend, she's made some changes. We're locked into the conference tournament. We're going to be, we could be anywhere one through four...so it's like it what's it matter if we're two or four... so let's starting changing it. Let's, let's get the mentality changing like that's not your spot, that's not, like you don't own the three spot. You know, you can play two, you can play four...like, we need to start making match ups to help us win. So that part of it she's started to change now, seven months in because it wasn't a big deal. Umm... and I think that's part of it. Like knowing what to change, how to change it.

Additionally, when the new coach's style of coaching was vastly different from the

previous coach's style, this appeared to have a negative impact on the coach turnover. Sasha, a swimmer, reported:

[...] but this year it felt like everything was completely [the new coach's] style and nothing like the year before or like... they didn't integrate anything from the past or like even from when [the previous coach] was here... so then it was just like there was like more headbutting on what practices should look like or should be.

\section{Athletes' Affective States}

Two primary themes emerged from the data that painted a picture of how the coach turnover might have impacted the student-athletes in regards to their affective states. Namely, 
INFLUENCE OF COACH TURNOVER ON STUDENT-ATHLETES

some coaches were able to foster a climate that resulted in the student-athletes experiencing more negative affect, while the student-athletes of other coaches experienced more positive affect, particularly concerning self-confidence.

Confidence. A number of the athletes noted the new coaches had a large impact on their confidence in particular. Mario, a football player, reported:

I think when [the new coach] came in he was just trying to establish a culture of winning in everything that we did in terms of the classroom, in the community, and then on the field. So, I think that was a big thing and I think that helped people's confidence realizing that like "winning" is not just an athletic thing. It's an everyday kind of thing you do with your life and it's all like subjective as well in terms of how you define "winning." And so I think confidence came from being involved in the community, and doing well in school, always going to class and all that stuff. And then working out harder than we've ever worked out. So, I think when he came in, he stressed that and then I think helped improve the confidence of players and right now it seems like the team is doing well.

On the other hand, Camille, a basketball player, said:

[...] they didn't really know how to communicate that confidence with us...so I think that has built up the past couple of years... but I think that's like kind of where the shakiness comes from...because they don't know everybody super well so it's like they don't know how to build each person's confidence level.

Anxiety. Numerous athletes also reported experiencing negative affective states following the coaching change. Especially immediately following the turnover, athletes reported experiencing anxiety, seemingly due to the coaching change and the uncertainties that came with 
INFLUENCE OF COACH TURNOVER ON STUDENT-ATHLETES

it. For example, Allen, an administrator, described the initial response he saw many of the athletes experience when they learned they were getting a new coach:

It's the anticipation of the pain, no different than getting a shot. The anticipation is always much worse than the shot, so when a coach leave, the initial reaction is...this is going to be the worst thing, I'm transferring. I came to [this university] to play for [the former coach] and now he's not going to...I'm leaving.

\section{Team Dynamics}

In addition to influencing the athletes' affective states, the coach turnover also affected a number of variables on the team level, namely, cohesion, communication, the coach-athlete relationship, and athlete leadership.

Cohesion. Several participants discussed how going through a coach turnover brought the athletes closer together. Brianna, a soccer player, described this increased cohesion as follows:

I think we kind of actually bonded through the team going through the [coaching] change. Because I think we relied more on each other and relied on like me talking to someone else rather than...like before we literally had people just going to like: 'I'm sorry I'm going to cuss here...' Like going and bitching to the coach and now it was more like within the team. We are closer because we're all dealing with the same thing rather than like some drama causing involving two people...Then it was an issue but like we all went through it together...we all had to make the same changes...so it kind of brought us closer together throughout that.

However, for some teams, coach turnover did not necessarily improve cohesiveness in the team, as Ana, a tennis player, pointed out: 
INFLUENCE OF COACH TURNOVER ON STUDENT-ATHLETES

[...] with [the former coach] we did all of these team bonding and team building activities... we did like a BBQ we had a full team retreat weekend where we did a bunch of stuff together that we just didn't do any of that with [the new coach]

Communication. Coaching turnover also affected team communication. Demarcus, a swimmer, described how his new coach improved team communication:

Yeah [the new coach is] a really good communicator of what is expected. In the past you kind of had to infer what they wanted you to do which I don't think it was the right strategy... So now it's everything is on the table... like 'this is what we're doing, you need to do it.'

On the other hand, some new coaches had a negative impact on team communication.

Sasha, a swimmer, said:

[the former coach] was really good at putting out like calendars and telling us when things are happening and [the new coach] sucked... She'll tell two people and be like tell the rest of the team and then five people aren't at practice and she's like why not...that was frustrating.

Coach-athlete relationship. A third theme that emerged pertaining to team dynamics was the coach-athlete relationship. Some new coaches were able to make changes that resulted in a positive relationship between the athletes and the new coach. Tiana, the new women's basketball coach, spoke about how her and her assistant coach devoted time to nurturing the coach-athlete relationship:

I thought we did a good job getting to know them as people besides the fact that they were...you know are [student-athletes] and wanting to perform well on the court. And I think that's really, really important...because then when you're asking them to do some 
INFLUENCE OF COACH TURNOVER ON STUDENT-ATHLETES

things differently they buy in and understand the process of how we teach and how we train, and I think that made it a lot easier.

However, other participants reported the new coaches did not seem to devote much time or effort to fostering a meaningful relationship with the athletes, resulting in a more difficult coach-athlete relationship. Jessica, a volleyball player, commented:

It was really hard for me to get used to these [new] coaches because...I feel like when they first got here they didn't really know how to talk to us... like they didn't know us as a person.

Athlete leadership. The last theme related to team dynamics pertained to how the coach turnover affected athlete leadership on the team. In most cases, participants reported that going through a coach turnover provided an opportunity for the athlete leaders to grow and further develop. For example, Mario, a football player, noted:

Yeah definitely in the absence of a...you know...prominent "head, head" of the table so to speak, you definitely had to assume that role. Yeah, so, it definitely gives you an extra push to do it and so I think it...it helps a lot cause then that transcends into whatever you know you want to do in the future as professionals. So, you know leadership transcends any field so it's umm... I think it's important and definitely gives you a little nudge to step up cause, if you don't, then no one is going to and then the team will kind of just kind of fall apart.

However, participants also reported that the student-athletes themselves suffered if the new coach failed to continue to support the development of the athlete leaders. Justin, a tennis player, said: 
INFLUENCE OF COACH TURNOVER ON STUDENT-ATHLETES

$[\ldots]$ there was this weird feeling on the team that nobody was really in charge of things and it was basic just the coach and then us...So, if anybody had a problem with the coach, they would end up just going straight to the coach or yelling at her or, you know, making a big deal out of it...And then she would react pretty negatively and yell or get mad at us and stuff... And it's like you need the bridge, you need the captains to be able to reasonably and go talk to the coach so you don't have kids you know freaking out during practice and yelling at her or whatever.

\section{Program Culture}

The final first-level theme that emerged from the data related to the overall environment. More specifically, participants discussed how the coach turnover influenced the entire team culture. On one hand, multiple administrators and student-athletes spoke of how the new coach was able to implement changes that resulted in a more positive team culture. Eli, a football player, described this culture change as follows:

$[\ldots]$ the new coach is way more involved with the community so there's a lot more exposure to the community and to the school... and people recognize us more differently because he pushes that social aspect, and the community service, and the schoolwork, and stuff like... so we have a better I guess light on the program in that sense.

On the other hand, athletics administrators in particular, discussed how taking a significant amount of time to fill the open coaching positions resulted in a negative impact on the sports program. Roger, said:

[...] holding coaching positions open affects the continuity, affects attitudes, affects recruiting, affects all of these things, so... that's harder for us when we look at neighbors 
INFLUENCE OF COACH TURNOVER ON STUDENT-ATHLETES

that are the bigger schools and they just... within a week, they're full, they're ready, they're rolling... we just can't do it.

\section{Discussion}

In the present study we explored the influence of coach turnover on athletes' affective states and team dynamics. Our inductive content analysis suggests that coach turnover might have both functional and dysfunctional effects on athletes' affective states and team dynamics, depending on the coaches' style and background characteristics. Furthermore, our findings revealed that coach turnover might have a systemic impact at the sports program level. The intricacies of these findings are elaborated upon next.

\section{Coaches' Characteristics and Turnover Success}

Coaches with different personalities and background characteristics (e.g., educational levels, nationalities) have different leadership styles that might be a better or worse fit for some athletes, teams, and cultures. To this extent, classic research in applied psychology has shown that coaches with different backgrounds and leadership approaches are more or less effective for expertise development depending on the level of the athlete or team, and the context at large (Bloom, 1985; Côté \& Gilbert, 2009; Coté, Saimela, Trudel, Baria, \& Russell, 1995). Moreover, different leadership styles (e.g., autocratic, democratic, transactional, transformational) have varying impacts on individuals' affective states, team dynamics, and communities at large (Boje, Haley, \& Saylors, 2016).

It follows that hiring a coach who is a "good fit" for a college sports team is paramount to ensure not only optimal performance but also satisfaction for the individual athletes, the team, and the college community. In this regard, fit theory has been used to inform hiring of personnel in companies of all types by adhering to a dynamic systems view of human performance, 
INFLUENCE OF COACH TURNOVER ON STUDENT-ATHLETES

whereby the impact of hiring and firing personnel is considered at a micro (i.e., individual), meso (e.g., departments and teams), and macro (e.g., company) level of analysis (see Hesketh \& Griffin, 2016). Importantly, our results suggest that the gender of the coach likely influences the success of the turnover process, especially in sports wherein gender stereotypes prevail. Steps to ensure gender equality, particularly educating collegiate athletes on gender topics, might be needed to prevent negative bias in athletes' perceptions of coaching effectiveness. Overall, hiring coaches that are a "good fit" across levels of analysis is important because coaches establish the motivational climate within the team, which in turn influences, positively or negatively, individuals' affective states (Duda et al., 2014).

\section{Coach Turnover and Athletes' Affective States}

Depending on the motivational climate fostered by the coach, athletes will experience more or less pleasant and functional affective states. In this regard, extant research suggests that a mastery-oriented motivational climate fosters the development of self-determined motivation and efficacy beliefs (for a review see Braithwaite et al., 2011). Conversely, coaches that promote an ego-oriented climate are more likely to generate negative and dysfunctional affective experiences among their athletes (Bortoli et al., 2014; Duda et al., 2014). Noteworthy, in addition to the content (e.g., pleasant or unpleasant) and functionality (e.g., functional or dysfunctional) of affective experiences, coaches' behaviors also influence the intensity of the affective responses experienced by athletes (Hanin, 2007). Put differently, coaches' might either help or hinder the athletes' ability to find their zone of optimal arousal and performance under pressure. This is not to suggest that coaches are "larger than life individuals," akin to great man theories of coaching and leadership (see Gavetti, 2011). Rather, our findings suggest that coach turnover benefits athletes' when it leads to the development of a more positive motivational climate. 
INFLUENCE OF COACH TURNOVER ON STUDENT-ATHLETES

However, if coach turnover leads to a worsening of motivational climate, athletes are more likely to experience negative affective states.

\section{Coach Turnover and Team Dynamics}

Our findings also suggest that coach turnover influences team processes, namely cohesion, collective efficacy, and peer leadership. Coaches with different backgrounds, personalities, and expertise will either promote or impede team dynamics. With respect to cohesion, our findings revealed that some coaches were perceived to be better at promoting social bonding and instrumental task connections than others. Indeed, it is well established that some coaches are better at developing relationships, whereas others focus more on promoting the pursuit of instrumental goals (Carron \& Eys, 2012). Ideally, optimal coach turnover will enhance both social and task cohesion, especially given the fact that high-performing teams are usually high on social and task cohesion (Filho, Gershgoren, Basevitch, \& Tenenbaum, 2014).

Our findings also revealed that different coaches were better sources of confidence to their teams than others. In fact, previous empirical and theoretical work has shown that modelling is a major source of collective efficacy in sport teams (Feltz, Short, \& Sullivan, 2008). In other words, confident coaches are, in principle, more likely to model efficacious behaviors to their teams. Coaches can also instill efficacy beliefs in their teams through verbal persuasion. That is, coaches who communicate more positively and confidently will likely enhance the morale of their teams. Importantly, collective efficacy is thought to be reciprocally linked to other team processes (see reciprocal determinism; Bandura, 1997). Therefore, coach turnover influences team dynamics as a whole rather than as a single team attribute. Coaches who enhance collective efficacy in their team are also likely to influence other team processes, such as cohesion and shared mental models, and peer leadership (Filho, Tenenbaum, \& Yang, 2014). 
INFLUENCE OF COACH TURNOVER ON STUDENT-ATHLETES

Our results suggest that coach turnover might either contribute to or hinder the development of peer leadership within a given team. For instance, more authoritarian coaches might be less willing to empower peer leaders. On the other hand, democratic coaches might be more likely to decentralize control by delegating on- and off-field responsibilities to their players. Furthermore, depending on how coaches' pick their captains, positive team dynamics might be either enhanced or diminished. For instance, some coaches may favor technical skill when choosing their captains, whereas other coaches might value experience or communication skills. Peer leaders are important in regulating the cognitive-affective-behavioral states of their fellow teammates (Filho, Gershgoren, Basevitch, Schinke, \& Tenenbaum, 2014). As such, coaches who promote the development of leadership skills among players are more likely to be beneficial to their teams and sport community at large.

\section{Coach Turnover and Program Culture}

A single individual can change an entire system. As has been said, “... the flutter of a butterfly's wing can ultimately cause a typhoon halfway around the world" (Chaos Theory). Put plainly, outstanding transformational leaders in politics, civil rights, and sports positively influence not only the individuals and teams they work with, but also local communities and society at large (Filho \& Rettig, 2016). That is, coach turnover might make or break a program in the long run. Therefore, it is crucial to involve various representatives of the athletic community (e.g., parents, fans, staff, and athletes) in the hiring of new coaches. Beyond recruitment, retention is also paramount in discussing coach turnover in sports. Coaches need to feel secure in their position and be given adequate time to implement new ideas and actions that may result in positive culture change at the community level. Hence, athletic departments that are satisfied with their coaching staff should make an effort to retain coaches by, for instance, putting in place 
INFLUENCE OF COACH TURNOVER ON STUDENT-ATHLETES

strategic measures to prevent coaching burnout (e.g., policies to ensure work-life balance and reduce excessive travelling; Olusoga \& Kenttä, 2017).

\section{Limitations, Future Research, and Applied Implications}

The present study represents a qualitative analysis of coach turnover in collegiate sports. As such, causal input-output relations on how coach turnover influences athletes, teams, and communities cannot be established. Rather, the findings presented and discussed herein carry descriptive value only. Further research, particularly quasi-experimental studies in real contexts (for an example, see Bortoli et al., 2014) are needed to advance understanding of the causal means-ends of coach turnover on individuals, teams, and contexts. Moreover, as a case study, generalizability of our findings is limited. To circumvent this limitation, larger studies combining data sets from different cases, research groups (i.e., multi-site studies), and countries should be carried out. In particular, we encourage studies in which coach turnover occurred during the season, as the present study included only coach turnover that took place out of season. Furthermore, quantitative analysis, particularly through longitudinal growth modelling assessments, is important to advance understanding of the immediate, mid-range, and long-term effects of coach turnover in sports. For instance, it is likely that the effect of coach turnover on other team processes, such as team mental models, can only be identified over time and through the use of quantitative measures. Additionally, it is important to consider that there are circumstances where a coach is not successful "on the field" (i.e., team is not winning) but still has a positive influence on the players' well-being and the community at large. Future studies embracing both objective and subjective measures of performance at the individual, team, and community level of analysis are needed to further illuminate this matter. In a similar vein, it is important to consider that the success of an incoming coach might depend on his/her 
INFLUENCE OF COACH TURNOVER ON STUDENT-ATHLETES

predecessor; for instance, replacing a successful coach might be easier or harder than an unsuccessful coach.

Despite the aforementioned limitations, our study advances research in coaching by contributing knowledge on the dynamic iterative effects of coach turnover in collegiate sports. Coach turnover is dynamic insofar that it can foster either functional or dysfunctional effects on individuals' affective states, team dynamics, and university environments. Both hiring and retaining effective coaches carries systemic implications in collegiate sports. Thus, practitioners might facilitate the positive impact of coach turnover by assessing whether the new coach is a "good fit" at the individual, team, and environmental level of analysis. Overall, gaining insight from the student-athletes, teams, and the athletic programs at large seems to be essential to ensure a successful coach turnover. 
INFLUENCE OF COACH TURNOVER ON STUDENT-ATHLETES

\section{References}

Audas, R., Dobson, S., \& Goddard, J. (2002). The impact of managerial changes on team performance in professional sports. Journal of Economics and Business, 54, 633-650.

Bandura, A. (1997). Self-efficacy: The exercise of control. New York, NY: WH Freeman/Times Books/Henry Holt.

Bloom, B. S., \& Sosniak, L. A. (1985). Developing talent in young people. New York, NY: Ballantine Books.

Boje, D. M., Haley, U. C., \& Saylors, R. (2016). Antenarratives of organizational change: The microstoria of Burger King's storytelling in space, time and strategic context. Human Relations, 69, 392-418.

Bortoli, L., Bertollo, M., Comani, S., \& Robazza, C. (2011). Competence, achievement goals, motivational climate, and pleasant psychobiosocial states in youth sport. Journal of Sports Sciences, 29, 171-180.

Bortoli, L., Bertollo, M., Filho, E., \& Robazza, C. (2014). Do psychobiosocial states mediate the relationship between perceived motivational climate and individual motivation in youngsters? Journal of Sports Sciences, 32, 572-582. DOI:

$10.1080 / 02640414.2013 .843017$

Braithwaite, R., Spray, C. M., \& Warburton, V. E. (2011). Motivational climate interventions in physical education: a meta-analysis. Psychology of Sport and Exercise, 12, 628-638. DOI: 10.1016/j.psychsport.2011.06.005

Brubaker, K. (2007). Coaching and teaching our athletes. Coach and Athletic Director, 77(3), 30-33. 
INFLUENCE OF COACH TURNOVER ON STUDENT-ATHLETES

Carron, A. V., \& Eys, M. A. (2012). Group dynamics in sport (4th ed.). Morgantown, WV: Fitness Information Technology.

Colvin, M., Blom, L., \& Bastin, C. (2012). The impact of season success on new coach-athlete relationships. Journal for the Study of Sports and Athletes in Education, 6, 311-324.

Coté, J., Saimela, J., Trudel, P., Baria, A., \& Russell, S. (1995). The coaching model: A grounded assessment of expert gymnastic coaches' knowledge. Journal of Sport and Exercise Psychology, 17, 1-17.

Côté, J., \& Gilbert, W. (2009). An integrative definition of coaching effectiveness and expertise. International Journal of Sports Science and Coaching, 4(3), 307-323.

Cunningham, G. B., Sagas, M., \& Ashley, F. B. (2001). Occupational commitment and the intent to leave the coaching profession: Differences according to race. International Review for the Sociology of Sport, 36, 131-148.

Davidsen, A. S. (2013). Phenomenological approaches in psychology and health sciences. Qualitative Research in Psychology, 10, 318-339.

Duda, J. L., Papaioannou, A. G., Appleton, P. R., Quested, E., \& Krommidas, C. (2014). Creating adaptive motivational climates in sport and physical education. In A. G. Papaioannou \& D. Hackfort (Eds.), Routledge companion to sport and exercise psychology: Global perspectives and fundamental concepts (pp. 544-558). New York, NY: Routledge.

Fabianic, D. (1994). Managerial change and organizational effectiveness in major league baseball: Findings for the eighties. Journal of Sport Behavior, 17, 135-147.

Feltz, D. L., Short, S. E., \& Sullivan, P. J. (2008). Self-efficacy in sport. Champaign, IL: Human Kinetics. 
INFLUENCE OF COACH TURNOVER ON STUDENT-ATHLETES

Filho, E., \& Rettig, J. (2016). Intergroup conflict management strategies from a Nobel Peace Laureate: The case of José Ramos-Horta. Basic and Applied Social Psychology, 38, 351361.

Filho, E., Gershgoren, L., Basevitch, I., Schinke, R., \& Tenenbaum, G. (2014). Peer leadership and shared mental models in a college volleyball team: A season long case study. Journal of Clinical Sport Psychology, 8, 184-203.

Filho, E., Gershgoren, L., Basevitch, I., \& Tenenbaum, G. (2014). Profile of high-performing college soccer teams: An exploratory multi-level analysis. Psychology of Sport \& Exercise, 15, 559-568. DOI: 10.1016/j.psychsport.2014.05.008

Filho, E., Tenenbaum, G., \& Yang, Y. (2014). Cohesion, team mental models, and collective efficacy: towards an integrated framework of team dynamics in sport. Journal of Sports Sciences, 33, 641-653. DOI: 10.1080/02640414.2014.957714

Gavetti, G. (2011). The new psychology of strategic leadership. Harvard Business Review, 89, $118-125$.

Gershgoren, L., Filho, E. M., Tenenbaum, G., \& Schinke, R. J. (2013). Coaching shared mental models in soccer: A longitudinal case study. Journal of Clinical Sport Psychology, 7, 293-312.

Glaser, B. G. (1965). The constant comparative method of qualitative analysis. Social Problems, $12,436-445$.

Goldstein, J. D., \& Iso-Ahola, S. E. (2006). Promoting sportsmanship in youth sports: Perspectives from sport psychology. Journal of Physical Education, Recreation, \& Dance, 77, 18-24. 
INFLUENCE OF COACH TURNOVER ON STUDENT-ATHLETES

Hanin, Y. L. (2007). Emotions in sport: current issues and perspectives. In G. Tenenbaum \& R. Eklund (Eds.), Handbook of sport psychology (3rd ed., pp. 31-58). Hoboken, NJ: Wiley.

Hesketh, B., \& Griffin, B. (2016). Person-environment fit. The encyclopedia of adulthood and aging.

Humphreys, B. R., Paul, R. J., \& Weinbach, A. P. (2016). Performance expectations and the tenure of head coaching: Evidence from NCAA football. Research in Economics, 70, 482-492.

Jackson, B., Knapp, P., \& Beauchamp, M. R. (2009). The coach-athlete relationship: A tripartite efficacy perspective. The Sport Psychologist, 23, 203-232.

Johnson, J. E., Blom, L. C., Judge, L. W., Lee, D., Pierce, D. A., \& Ridley, M. J. (2013). The impact of football bowl subdivision head coaching changes on NCAA academic progress rate. Journal of Issues in Intercollegiate Athletics, 6, 131-154.

Johnson, J. E., Wessel, R. D., \& Pierce, D. A. (2012). The influence of selected variables on NCAA Academic Progress Rate. Journal of Issues in Intercollegiate Athletics, 5, 149171.

Jowett, S. (2005). The coach-athlete partnership. The Psychologist, 18, 412-415.

Jowett, S. (2009). Factor structure and criterion-related validity of the metaperspective version of the coach-athlete relationship questionnaire (CART-Q). Group Dynamics: Theory, Research, and Practice, 13, 163- 177.

Jowett, S., \& Ntoumanis, N. (2004). The Coach-Athlete Relationship Questionnaire (CART-Q): Development and initial validation. Scandinavian Journal of Medicine and Science in Sports, 14, 245-257. 
INFLUENCE OF COACH TURNOVER ON STUDENT-ATHLETES

Jowett, S., Paull, G., \& Pensgaard, A. M. (2005). Coach-athlete relationship. In J. Taylor \& G. S. Wilson (Eds.) Applying sport psychology: Four perspectives (pp. 153-170). Champaign, IL: Human Kinetics.

Lattman, M. (2008). The role of junior college coaches: More than the sport. Coach and Athletic Director, 77, 110-112.

Mitchell, T. R., Holtom, B. C., \& Lee, T. W. (2001). How to keep your best employees: developing an effective retention policy. Academy of Management Executive, 15, 96-108.

NCAA (January, 2011). Examining the student-athlete experience through the NCAA GOALS and SCORE studies. Presentation made at the NCAA National Convention, San Antonio, TX. Retrieved from http://www.ncaa.org/wps/wcm/connect/public/ncaa/pdfs/2011/examining+the+ student+athlete+experience+through+the+ncaa+goals+and+score+studies

Nicholls, J. G. (1992). "The general and the specific in the development and expression of achievement motivation", in Motivation in Sport and Exercise, ed. G. C. Roberts (Champaign, IL: Human Kinetics), 31-56.

Olusoga, P., \& Kenttä, G. (2017). Desperate to quit: A narrative analysis of burnout and recovery in high performance sports coaching. The Sport Psychologist, 1-32.

Pate, J. P., Stokowski, S, E., \& Hardin, R. (2011). Third time's a charm: The case of Tennessee's four junior football players who endured three different head coaches in three seasons. Journal of Issues in Intercollegiate Athletics, 4, 354-369.

Patton, M. Q. (2002). Qualitative research and evaluation methods ( $3^{\text {rd }}$ ed.). Thousand Oaks, CA: Sage Publications.

Raedeke, T. D., Warren A. H., \& Granzyk, T. L. (2002). Coaching commitment and turnover: A 
INFLUENCE OF COACH TURNOVER ON STUDENT-ATHLETES

comparison of current and former coaches. Research Quarterly for Exercise and Sport, 73, 73-86.

Ryan, T. D., \& Sagas, M. (2009). Relationship between pay satisfaction, work-family conflict, and coaching turnover intentions. Team Performance Management, 15, 128-140.

Shipherd, A. M., Basevitch, I., Barcza-Renner, K. B., \& Siwatu, K. O. (2014). Development and evaluation of a team building intervention with a U.S. collegiate rugby team: A mixed methods approach. Journal of Multidisciplinary Research, 6, 31-48.

Smith, J. A., \& Osborn, M. (2015). Interpretative phenomenological analysis. In J. A. Smith (Ed.), Qualitative psychology: A practical guide to research methods (pp. 53-80). Thousand Oaks, CA: Sage Publications.

Solomon, G. B., Striegel, D. A., Eliot, J. F., Heon, S. N., Maas, J. L., \& Wayda, V. K. (1996). The self-fulfilling prophecy in college basketball: Implications for effective coaching. Journal of Applied Sport Psychology, 8, 44-59.

Tenenbaum, G., Basevitch, I., Gershgoren, L., \& Filho, E. (2013). Emotions-decision-making in sport: Theoretical conceptualization and experimental evidence. International Journal of Sport and Exercise Psychology, 11, 151-168.

Turman, P. D. (2008). Coaches' immediacy behaviors as predictors of athletes' perceptions of satisfaction and team cohesion. Western Journal of Communication, 72, 162-179.

Weiss, M. R., \& Sisley, B. L. (1984). Where have all the coaches gone? Sociology of Sport Journal, 1, 332-347.

White, P., Persad, S., \& Gee, C. J. (2007). The effect of mid-season coach turnover on team performance: The case of the national hockey league (1989-2003). International Journal of Sports Science \& Coaching, 2, 143-152. 
INFLUENCE OF COACH TURNOVER ON STUDENT-ATHLETES

Winthrop Intelligence. (2013, September 2). Division-I coaching turnover trends. Retrieved from http://winthropintelligence.com/2013/09/02/coaching-turnover-trends/ 